\title{
A ROOT OF UNITY OCCURRING IN PARTITION THEORY
}

\author{
PETER HAGIS, JR.
}

ABSTRACT. In this paper a new representation is found for the root of unity occurring in the well-known transformation equation of the generating function for $p(n)$, the number of partitions of the positive integer $n$.

Let $p(n)$ denote the number of partitions of the positive integer $n$. In the transformation equation for the generating function of $p(n)$ (see [1] and [3]) there occurs a certain root of unity which we shall denote by $\omega(h, k)$. Here $k$ is a positive integer and $h$ is an integer coprime to $k . \omega(h, k)$ also appears in the exponential sums $A_{k}(n)$ which occur in the infinite series represention of $p(n)$ due to Rademacher [3]. It should be mentioned, however, that a formula for $A_{k}(n)$ has been found by Selberg which does not depend on $\omega(h, k)$ (see [5] and [6]).

In [1] it was shown by Hardy and Ramanujan that

$$
\begin{aligned}
\omega(h, k)=(-h \mid k) \exp \{-\pi i((k-1) / 4 & \\
& \left.\left.+\left(k^{2}-1\right)\left(2 h+H-h^{2} H\right) / 12 k\right)\right\}
\end{aligned}
$$

if $k$ is odd, and

$$
\begin{aligned}
\omega(h, k)=(-k \mid h) \exp \{-\pi i((2-h k & -h) / 4 \\
& \left.\left.+\left(k^{2}-1\right)\left(2 h+H-h^{2} H\right) / 12 k\right)\right\}
\end{aligned}
$$

if $k$ is even. Here, and in the sequel, $(a \mid b)$ is the Jacobi symbol while $H$ is any solution of the congruence $h H \equiv 1(\bmod k)$. In [2] Rademacher showed that $\omega(h, k)=\exp \{\pi i s(h, k)\}$ where $s(h, k)$ is a Dedekind sum defined by $s(h, k)=\sum_{u=1}^{k}((u / k))((h u / k))$ with $((x))$ $=0$ if $x$ is an integer and $((x))=x-[x]-1 / 2$ otherwise.

The purpose of the present note is to present still another representation of $\omega(h, k)$ which appears to be somewhat simpler to handle in computations than those just stated. Thus, we shall prove the following

\section{THEOREM. If $k$ is odd then}

Received by the editors March 20, 1970.

AMS 1969 subject classifications. Primary 1048.

Key words and phrases. Partitions, generating function, transformation equation, roots of unity. 


$$
\omega(h, k)=(h \mid k) i^{(k-1) / 2} \exp \left\{2 \pi i q\left(h-h^{\prime}\right) / g k\right\} ;
$$

if $k$ is even then

$$
\omega(h, k)=(k \mid h) i^{b(k+1) / 2} \exp \left\{2 \pi i q\left(h-h^{\prime}\right) / g k\right\} .
$$

If $J=(k, 3)$ then $g=J$ or $8 J$ according as $k$ is odd or even. $h^{\prime}$ is any solution of $h h^{\prime} \equiv-1(\bmod g k)$, and $q$ is any solution of $24 q / g=1$ $(\bmod g k)$. In $(2) h^{\prime} \equiv b(\bmod 8)$, and the branch of $i^{b(k+1) / 2}$ is that corresponding to the principal value of $\log z$.

Our proof is based on four lemmas concerning the Dedekind sums $s(h, k)$. These are essentially Theorems $17,18,19$ in [4].

LEMMA $1.12 k s(h, k) \equiv 0(\bmod 3)$ if and only if $J=1$.

LEMMA 2. $12 k s(h, k) \equiv h-h^{\prime}(\bmod J k)$.

Lemma 3. If $k$ is odd, then $12 k s(h, k) \equiv k+1-2(h \mid k)(\bmod 8)$.

Lemma 4. If $k=2^{a} K, a>0$ and $K$ odd, then

$$
12 k s(h, k) \equiv h-h^{\prime}-h^{\prime} k^{2}-3 h^{\prime} k+6 h^{\prime} k(k \mid h)\left(\bmod 2^{a+3}\right) .
$$

Now suppose first that $k$ is odd and let $f=24 / J$. Then

$$
12 k s(h, k) \equiv 9 k+9+6(h \mid k) \quad(\bmod f) .
$$

For if $f=8$ then (3) follows immediately from Lemma 3, while if $f=24$ then (3) follows from Lemmas 1,3 and the Chinese Remainder Theorem.

If we define the integers $q$ and $r$ by the congruences

$$
f q \equiv 1 \quad(\bmod J k), \quad k r \equiv 1 \quad(\bmod f),
$$

then it follows from Lemma 2, (3), (4) that

$$
12 k s(h, k) \equiv k r(9 k+9+6(h \mid k))+f q\left(h-h^{\prime}\right)(\bmod 24 k) .
$$

Therefore,

$$
\begin{aligned}
\omega(h, k) & =\exp \{2 \pi i(12 k s(h, k) / 24 k)\} \\
& =\exp \left\{2 \pi i\left(r(9 k+9+6(h \mid k)) / 24+q\left(h-h^{\prime}\right) / J k\right)\right\} .
\end{aligned}
$$

Since $9=-9+18$ and since $2 r(18+6(h \mid k) / 24$ is even or odd according as $(h \mid k)=1$ or $(h \mid k)=-1$, respectively, we see that

$$
\omega(h, k)=(h \mid k) \exp \left\{2 \pi i q\left(h-h^{\prime}\right) / J k\right\} \exp \{3 \pi i r(k-1) / 4\} .
$$

From (4) we have $r \equiv k(\bmod 8)$, and since $\exp \{3 \pi i k(k-1) / 4\}$ $=i^{(k-1) / 2}$ the proof of $(1)$ is complete.

If $k$ is even let $f=24 / 8 \mathrm{~J}$. Then 


$$
12 k s(h, k) \equiv 0 \quad(\bmod f)
$$

is immediate if $f=1$ and follows from Lemma 1 if $f=3$. From Lemmas 2 and 4 we have

$$
12 k s(h, k) \equiv h-h^{\prime}\left(1+k^{2}+3 k-6 k(k \mid h)\right) \quad(\bmod 8 J k),
$$

and if $q$ is defined by the congruence

$$
f q \equiv 1 \quad(\bmod 8 J k)
$$

we see from (5) and (6) that

$$
12 k s(h, k) \equiv f q\left(h-h^{\prime}\left(1+k^{2}+3 k-6 k(k \mid h)\right)\right) \quad(\bmod 24 k) .
$$

Therefore,

$$
\begin{aligned}
\omega(h, k) & =\exp \{2 \pi i(12 k s(h, k) / 24 k)\} \\
& =\exp \left\{2 \pi i q\left(h-h^{\prime}\left(1+k^{2}+3 k-6 k(k \mid h)\right)\right) / 8 J k\right\} .
\end{aligned}
$$

Since $3 k \equiv-3 k-18 k(\bmod 8 J k)$, and since $2 q h^{\prime}(18 k+6 k(k \mid h)) / 8 J k$ is even or odd according as $(k \mid h)=1$ or $(k \mid h)=-1$, we see that

(8) $\omega(h, k)=(k \mid h) \exp \left\{2 \Pi i q h^{\prime}(3-k) / 8 J\right\} \exp \left\{2 \Pi i q\left(h-h^{\prime}\right) / 8 J k\right\}$.

If $J=1$ then $f=3$, and from $(7)$ we have $q \equiv 3(\bmod 8)$ so that $q h^{\prime}(3-k) \equiv h^{\prime}(9-3 k) \equiv h^{\prime}(1+k)(\bmod 8)$. If $J=3$ then $f=1$, and from (7) we have $q \equiv 9(\bmod 8)$ so that $q h^{\prime}(1-k / 3) \equiv h^{\prime}(9-3 k)$ $\equiv h^{\prime}(1+k)(\bmod 8)$. Since $\exp \left\{2 \pi i h^{\prime}(1+k) / 8\right\}=\exp \{\pi i b(1+k) / 4\}$ if $h^{\prime} \equiv b(\bmod 8)$ we see that (2) follows from (8) and the proof of the theorem is complete.

We remark in closing that although $\omega(h, k)$ is almost always referred to in the literature as a $24 k$ th root of unity it is obvious from our theorem that it is also a $12 k$ th root of unity.

ADDED IN PROOF. Since this paper was accepted for publication it has been brought to the author's attention that Professor T. M. Apostol observed that $\omega(h, k)$ is a $12 k$ th root of unity in his doctoral dissertation (University of California at Berkeley, 1948). His observation, however, was not published.

AckNowledgement. The author would like to thank the referee for several helpful suggestions.

\section{REFERENCES}

1. G. H. Hardy and S. Ramanujan, Asymptotic formulae in combinatorial analysis, Proc. London Math. Soc. (2) 17 (1918), 75-115.

2. H. Rademacher, Zur Theorie der Modulfunktionen, J. Reine Angew. Math. 167 (1931), 312-336. 
3. H. Rademacher, On the partition function $p(n)$, Proc. London Math. Soc. (2) 43 (1937), 241-254.

4. H. Rademacher and A. Whiteman, Theorems on Dedekind sums, Amer. J. Math. 63 (1941), 377-407. MR 2, 249.

5. H. Rademacher, On the Selberg formula for $A_{k}(n)$, J. Indian Math. Soc. 21 (1957), 41-55. MR 19, 1163.

6. A. Whiteman, A sum connected with the series for the partition function, Pacific J. Math. 6 (1956), 159-176. MR 18, 195.

Temple University, Philadel.phia, Pennsylvania 19122 